\title{
Nonlinear Magnetic Induction by Helical Motion in a Liquid Sodium Turbulent Flow
}

\author{
F. Pétrélis, ${ }^{1}$ M. Bourgoin, ${ }^{2}$ L. Marié,${ }^{3}$ J. Burguete, ${ }^{3, *}$ A. Chiffaudel, ${ }^{3}$ F. Daviaud, ${ }^{3}$ S. Fauve, ${ }^{1}$ P. Odier, ${ }^{2}$ and J.-F. Pinton ${ }^{2}$ \\ ${ }^{1}$ Laboratoire de Physique Statistique de l'Ecole Normale Supérieure, CNRS UMR 8550, 24 Rue Lhomond, \\ 75231 Paris Cedex 05, France \\ ${ }^{2}$ Laboratoire de Physique de l'Ecole Normale Supérieure de Lyon, CNRS UMR 5672, 47 allée d'Italie, \\ 69364 Lyon Cedex 07, France \\ ${ }^{3}$ Service de Physique de l'Etat Condensé, Direction des Sciences de la Matière, CEA-Saclay, CNRS URA 2464, \\ 91191 Gif-sur-Yvette cedex, France
}

(Received 7 December 2001; revised manuscript received 14 January 2003; published 2 May 2003)

We report an experimental study of the magnetic field $\vec{B}$ induced by a turbulent swirling flow of liquid sodium submitted to a transverse magnetic field $\vec{B}_{0}$. We show that the induced field can behave nonlinearly as a function of the magnetic Reynolds number, $R_{m}$. At low $R_{m}$, the induced mean field along the axis of the flow, $\left\langle B_{x}\right\rangle$, and the one parallel to $\vec{B}_{0},\left\langle B_{y}\right\rangle$, first behave like $R_{m}^{2}$, whereas the third component, $\left\langle B_{z}\right\rangle$, is linear in $R_{m}$. The sign of $\left\langle B_{x}\right\rangle$ is determined by the flow helicity. At higher $R_{m}, \vec{B}$ strongly depends on the local geometry of the mean flow: $\left\langle B_{x}\right\rangle$ decreases to zero in the core of the swirling flow but remains finite outside. We compare the experimental results with the computed magnetic induction due to the mean flow alone.

Transport and amplification of a magnetic field by a flow of an electrically conducting fluid is a fundamental process in astrophysics at the planetary, stellar, and galactic scales [1], as well as in laboratory plasmas, where it has been observed for a long time that a toroidal magnetic field can be sustained by applying a toroidal electric field [2]. Induced magnetic fields orthogonal to the applied ones have also been observed in flows of liquid metals: generation of a toroidal field from an axial one by differential rotation (the " $\omega$ effect") [3-5], and generation of an electric current parallel to the applied magnetic field (the " $\alpha$ effect") [6]. These induction effects are the key mechanisms of most astrophysical and geophysical dynamo models [7-10], as well as in the recent experimental observations of self-generation of a magnetic field by a flow of liquid sodium [11]. These experiments involve flows with geometrical constraints that are chosen in order to maximize the efficiency of the dynamo effect. Several groups are also trying to achieve self-generation of a magnetic field in turbulent flows without, or with fewer, geometrical constraints, in order to study situations that are closer to astrophysical or geophysical models [12]. It is thus of primary interest to study induction effects in fully developed turbulent flows. It is known that turbulent fluctuations enhance the effective resistivity [13] but other antagonistic effects such as the turbulent $\alpha$ effect [8,9] or " $\beta$ effect" [14] have been predicted but not experimentally observed so far.

We have measured the induced magnetic field $\vec{B}$ generated by a turbulent von Kármán swirling flow of liquid sodium submitted to a transverse external magnetic field $\vec{B}_{0}$ (see Fig. 1). The sodium flow is operated in a loop that has been described elsewhere together with the details of the experimental setup [5]. In this study, the flow is driven by rotating one of the two disks of radius $R$ located at position (1) or (2) in a cylindrical vessel, $410 \mathrm{~mm}$ in inner diameter and $400 \mathrm{~mm}$ in length. In most experiments presented here, we use disks of radius $R=150 \mathrm{~mm}$, fitted with eight straight blades of height $h=10 \mathrm{~mm}$ driven at a rotation frequency up to $f=30 \mathrm{~Hz}$. Four baffles, $20 \mathrm{~mm}$ in height, have been mounted on the cylindrical vessel inner wall, parallel to its axis. A turbulent swirling flow with an integral Reynolds number, $\operatorname{Re}=2 \pi R^{2} f / \nu$, up to $3 \times 10^{6}$ is driven by the rotating disk. The mean flow has the following characteristics: the fluid is ejected radially outward by the disk; this drives an axial flow toward the disk along its axis and a recirculation in the opposite direction along the cylinder lateral boundary. The baffles inhibit the azimuthal velocity of the recirculating flow and thus prevent a global rotation of the fluid.

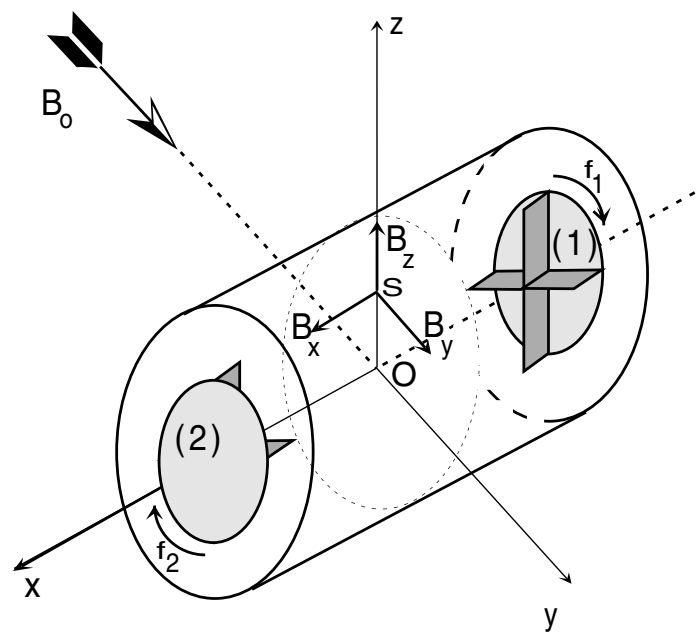

FIG. 1. Geometry of the experimental setup. The flow is generated by rotating only one disk either at position (1) or (2). The magnetic field is measured at position $S$. 
In some experiments, we have used a disk of radius $R=$ $190 \mathrm{~mm}$, fitted with 16 curved blades of height $h=$ $40 \mathrm{~mm}$, with or without the lateral baffles in order to observe the effect of a stronger azimuthal flow. Without baffles, we have for the maximum velocity of the mean flow $V_{M} / 2 \pi f R \approx 0.39$ for $R=150 \mathrm{~mm}$ and 0.58 for $R=$ $190 \mathrm{~mm}$. Two coils generate a nearly homogeneous magnetic field $\vec{B}_{0}$, perpendicular to the cylinder axis (see Fig. 1). The three components of the field induced by the flow are measured with a 3D Hall probe, located $200 \mathrm{~mm}$ away from the disk in the plane perpendicular to $\vec{B}_{0}$ and containing the rotation axis. The probe distance from the rotation axis is adjustable $(z=42,100,150 \mathrm{~mm})$.

The equations governing the magnetic field $\vec{B}_{0}+$ $\vec{B}(\vec{r}, t)$, where $\vec{B}(\vec{r}, t)$ is the magnetic field generated by the flow in the presence of the applied field $\vec{B}_{0}$, are in the MHD approximation,

$$
\begin{gathered}
\vec{\nabla} \cdot \vec{B}=0, \\
\frac{\partial \vec{B}}{\partial t}=\vec{\nabla} \times\left(\vec{V} \times\left(\vec{B}+\vec{B}_{0}\right)\right)+\frac{1}{\mu_{0} \sigma} \Delta \vec{B},
\end{gathered}
$$

where $\vec{V}(\vec{r}, t)$ is the velocity field, $\mu_{0}$ is the magnetic permeability of vacuum, and $\sigma$ is the fluid electric conductivity. The reaction of the magnetic field on the flow is characterized by the ratio of the Lorentz force to the characteristic pressure forces driving the flow. This is measured by the interaction parameter, $N=\sigma R B_{0}^{2} /$ $\rho V_{M}$, where $\rho$ is the fluid density. The maximum field amplitude being $B_{0}=12 \mathrm{G}, N$ is in the range $10^{-4}-10^{-2}$, thus the effect of the magnetic field on the flow is negligible. This has been checked directly by measuring $\vec{B}$ as a function of $\vec{B}_{0}$ at a constant driving of the flow. We calculate the mean induced field $\langle\vec{B}\rangle$ where $\langle\cdot\rangle$ stands for the average in time, as well as its rms fluctuations in time, $\vec{B}_{\text {rms }}$. Both vary linearly with $B_{0}$, thus showing that the modification of the velocity field $\vec{V}$ in Eq. (2) can be neglected [5]. Thus, the only relevant dimensionless parameters of our experiments are the magnetic Prandtl number, $P_{m}=\mu_{0} \sigma \nu \approx 10^{-5}$, where $\nu$ is the kinematic viscosity, and the magnetic Reynolds number, $R_{m}=$ $2 \pi \mu_{0} \sigma R^{2} f$, which is proportional to the rotation frequency $f$ and has been varied up to 40 for radius of the disks $R=150 \mathrm{~mm}$ (55 for $R=190 \mathrm{~mm}$ ).

The three components of the mean magnetic field $\vec{B}_{0}+\langle\vec{B}(\vec{r})\rangle$, at $z=100 \mathrm{~mm}$ above the rotation axis, are displayed in Fig. 2 as a function of the rotation frequency. We observe that when the rotation of the disk is reversed, $f \rightarrow-f$, we get approximately $\left(\left\langle B_{x}\right\rangle,\left\langle B_{y}\right\rangle,\left\langle B_{z}\right\rangle\right) \rightarrow$ $\left(-\left\langle B_{x}\right\rangle,\left\langle B_{y}\right\rangle,-\left\langle B_{z}\right\rangle\right)$. When disk (2) is rotated instead of (1) but keeping $f$ unchanged, we get $\left(\left\langle B_{x}\right\rangle,\left\langle B_{y}\right\rangle,\left\langle B_{z}\right\rangle\right) \rightarrow$ $\left(-\left\langle B_{x}\right\rangle,\left\langle B_{y}\right\rangle,\left\langle B_{z}\right\rangle\right)$ (note that the measurements of $\vec{B}$ are performed in the midplane between the two disks). Assuming that the swirling flow has not broken the symmetries of the driving configuration, the above trans-

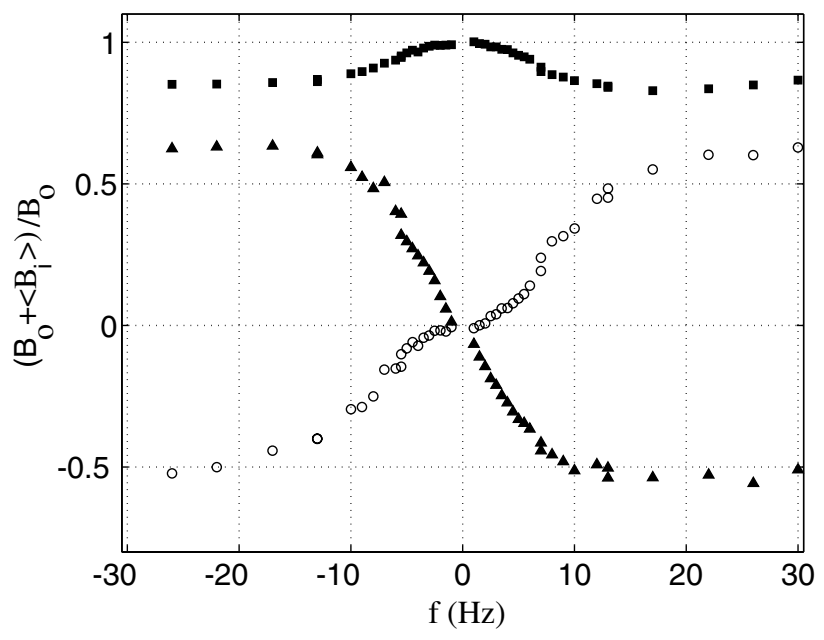

FIG. 2. Components of the total mean magnetic field as a function of the rotation frequency of disk (2). The disk radius is $R=150 \mathrm{~mm}$ with straight blades. Four baffles are mounted on the inner wall of the cylindrical vessel. The magnetic field is measured at $z=100 \mathrm{~mm}$. [(○) $\frac{\left\langle B_{x}\right\rangle}{B_{o}} ;$ (口) $\left.\frac{B_{o}+\left\langle B_{y}\right\rangle}{B_{o}} ;(\boldsymbol{\Delta}) \frac{\left\langle B_{z}\right\rangle}{B_{o}}\right]$.

formations of the field components can be understood using the following symmetry transformations.

(i) The symmetry with respect to the vertical plane perpendicular to $\vec{B}_{0}, x 0 z$, shows that if the disk is rotated in the opposite way, $f \rightarrow-f$, we get $\left(\left\langle B_{x}\right\rangle,\left\langle B_{y}\right\rangle,\left\langle B_{z}\right\rangle\right) \rightarrow$ $\left(-\left\langle B_{x}\right\rangle,\left\langle B_{y}\right\rangle,-\left\langle B_{z}\right\rangle\right)(\vec{B}$ is a pseudovector).

(ii) The symmetry with respect to the vertical plane parallel to $\vec{B}_{0}, y 0 z$, followed by the transformation $\vec{B}_{0} \rightarrow-\vec{B}_{0}$, shows that when we rotate disk (2) instead of disk (1) without changing the sign of $f$, we get $\left(\left\langle B_{x}\right\rangle,\left\langle B_{y}\right\rangle,\left\langle B_{z}\right\rangle\right) \rightarrow\left(-\left\langle B_{x}\right\rangle,\left\langle B_{y}\right\rangle,\left\langle B_{z}\right\rangle\right)$.

The induced field component $\left\langle B_{y}\right\rangle$ at $z=100 \mathrm{~mm}$ above the rotation axis is opposed to $\vec{B}_{0}$ and increases in amplitude, thus the total field along $\vec{B}_{0}$ decreases as $R_{m}$ is increased. This expulsion of a transverse magnetic field from eddies is well documented, both theoretically [15] and experimentally [16]. The expulsion is stronger close to the axis of the cylinder $(z=42 \mathrm{~mm})$. On the contrary, closer to the cylinder lateral boundary $(z=150 \mathrm{~mm})$, the field increases with $R_{m}$. Thus, the field is expelled from the core of the swirling flow and concentrates at its periphery. The components of the field induced perpendicular to $\vec{B}_{0}$ at $z=100 \mathrm{~mm}$ both increase in amplitude from zero, reach a maximum, and then saturate when $R_{m}$ is increased further. The large $R_{m}$ behavior depends on $z$ as shown for $\left\langle B_{x}\right\rangle$ in Fig. 3. $\left\langle B_{x}\right\rangle$ vanishes at large $R_{m}$ in the core of the swirling flow $(z=42 \mathrm{~mm})$, whereas it saturates outside $(z=100$ and $150 \mathrm{~mm})$. Figure 3 also shows that for fixed $R_{m},\left\langle B_{x}\right\rangle$ increases with the distance to the cylinder axis in the range $42<z<150 \mathrm{~mm}$. We can show that it should vanish for $z=0$ : indeed, the rotation of angle $\pi$ around the $x$ axis followed by the transformation $\vec{B}_{0} \rightarrow-\vec{B}_{0}$, which implies $\vec{B} \rightarrow-\vec{B}$, gives $\left\langle B_{x}(x, 0, z)\right\rangle=-\left\langle B_{x}(x, 0,-z)\right\rangle$. 


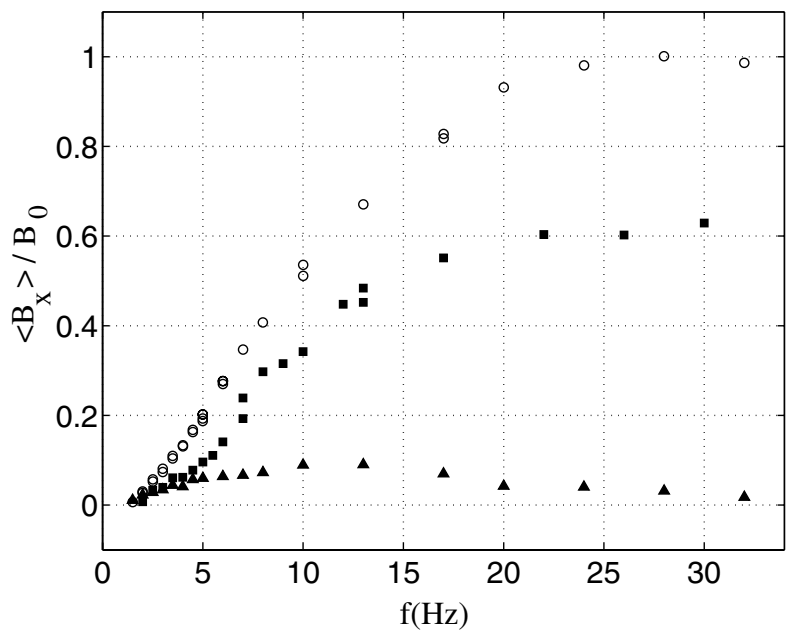

FIG. 3. Axial mean component of the induced magnetic field as a function of the rotation frequency of disk (2) for different depths $z:(\boldsymbol{\Delta}) z=42 \mathrm{~mm},(\boldsymbol{\square}) z=100 \mathrm{~mm},(\bigcirc) z=150 \mathrm{~mm}$. Same experimental configuration as in Fig. 2.

As shown in Fig. $4,\left\langle B_{x}\right\rangle$ and $\left\langle B_{z}\right\rangle$ do not scale in the same way at small rotation frequency, i.e., at small $R_{m}$. The amplitude of the vertical component $\left\langle B_{z}\right\rangle$ increases linearly, whereas the axial component $\left\langle B_{x}\right\rangle$ increases quadratically with $R_{m}$. Indeed, at the location of the measurements, near $z=100 \mathrm{~mm}$, we observe $\left\langle B_{x}\right\rangle \propto\left\langle B_{z}\right\rangle^{2}$ and $\left\langle B_{y}\right\rangle \propto\left\langle B_{z}\right\rangle^{2}$, roughly up to $f=10 \mathrm{~Hz}$ (see Fig. 4).

Writing $\vec{B}(\vec{r}, t)=\langle\vec{B}(\vec{r})\rangle+\vec{b}(\vec{r}, t)$, where $\vec{b}$ is the fluctuating part of the magnetic field, and similarly for $\vec{V}$, we get from Eq. (2) for the mean induced field

$$
-\frac{1}{\mu_{0} \sigma} \Delta\langle\vec{B}\rangle=\vec{\nabla} \times\left(\langle\vec{V}\rangle \times \vec{B}_{0}+\langle\vec{V}\rangle \times\langle\vec{B}\rangle+\langle\vec{v} \times \vec{b}\rangle\right) .
$$

When the magnetic Reynolds number is small, the first source term on the right hand side of Eq. (3) is the dominant one, and we usually get the $i$ th component of the mean induced field $\left\langle B_{i}\right\rangle \propto R_{m} B_{0}$. This is the case for the vertical component $\left\langle B_{z}\right\rangle$, which is mainly generated by the interaction of the toroidal velocity field with $\vec{B}_{0}$. The sign of $\left\langle B_{z}\right\rangle$ is thus given by the one of $f$.

However, we emphasize that both $\left\langle B_{x}\right\rangle$ and $\left\langle B_{y}\right\rangle$ behave quadratically at low $R_{m}$ (at least at the location of our measurements). We have thus probed the nonlinear source terms of Eq. (3). As shown above, $\left\langle B_{x}(x, 0, z)\right\rangle=$ $-\left\langle B_{x}(x, 0,-z)\right\rangle$, and $\left\langle B_{x}\right\rangle$ is generated by a current density parallel to $\vec{B}_{0}$. Its sign is changed by the two transformations (i) and (ii) described above and is thus determined by the flow helicity, $h=\overrightarrow{\vec{V} \cdot(\vec{\nabla} \times \vec{V})}$ where the overbar stands for the spatial average. One can easily check that $\left\langle B_{x}\right\rangle$ changes sign under any symmetry with respect to a plane containing the rotation axis, just as does the pseudoscalar $h$. Although the terminology $\alpha$ effect is usually
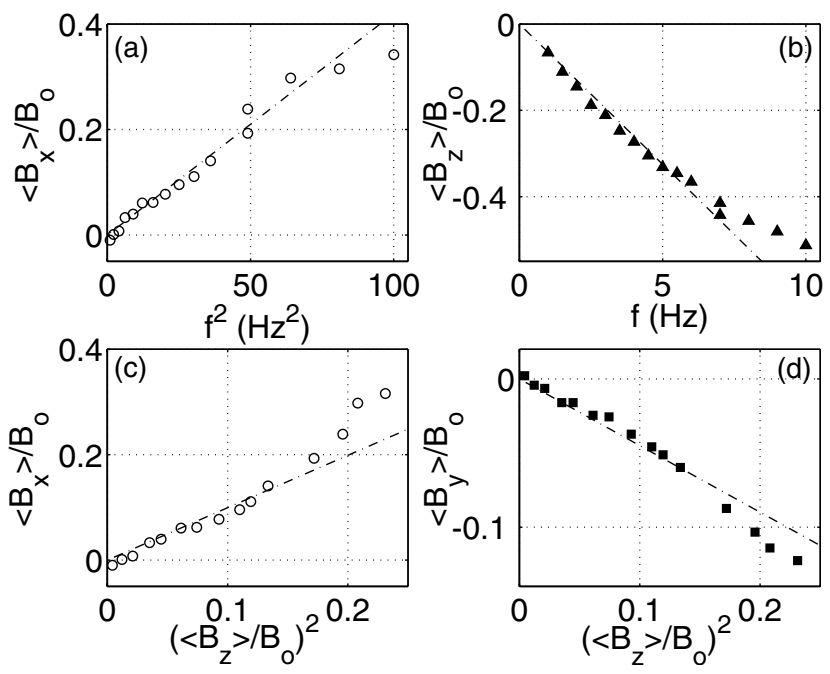

FIG. 4. (a),(b) Axial and vertical mean components of the induced magnetic field as a function of the rotation frequency. (c),(d) Axial and transverse mean components of the induced magnetic field as a function of the square of the vertical one, for $f<10 \mathrm{~Hz}$. Same experimental configuration as in Fig. 2 $(z=100 \mathrm{~mm})$.

restricted to configurations involving scale separation, whereas our experiment does not, we do observe the generation of a current parallel to $\vec{B}_{0}$ by a cyclonic eddy as described qualitatively by Parker [7]. In addition, its magnitude increases like $R_{m}^{2}$ at low $R_{m}$, and its sign is determined by the flow helicity, as in the case of the $\alpha$ effect.

When $R_{m}$ is increased, $\left\langle B_{x}\right\rangle$ seems to saturate for $z=$ $100 \mathrm{~mm}$ (see Fig. 2). Closer to the rotation axis $(z=$ $42 \mathrm{~mm}$ ), it reaches a maximum and then decreases roughly to zero when $f$ is increased up to $30 \mathrm{~Hz}$. This traces back to the expulsion of $\vec{B}_{0}$ from the core of the swirling flow. However, we emphasize that the effect of the expulsion on the induced fields is not straightforward. Indeed, $\left\langle B_{z}\right\rangle$ does not vanish but saturates to a finite value at high frequency, even close to the rotation axis. Induction mechanisms being nonlocal, the expulsion of $\overrightarrow{\boldsymbol{B}}_{0}$ from the core of the flow does not necessary imply that the induced fields all decrease to zero.

It is, however, possible to enhance the effect of the expulsion by removing the baffles from the cylindrical vessel inner wall, thus allowing global rotation of the flow. Then all the components of the induced field measured $100 \mathrm{~mm}$ away from the rotation axis decrease almost to zero at large $R_{m}$ (see Fig. 5 and compare with Fig. 2 where they stay finite). We thus observe that the axial field decreases to zero at large $R_{m}$ when the azimuthal flow is large enough. A similar effect has been recently computed by Rädler et al. in the case of the Roberts flow [17]. These authors have shown by computing terms higher than the second order in $R_{m}$ that the $\alpha$ effect reaches a maximum and then tends to zero when $R_{m}$ is increased further (compare their Fig. 3 with our Fig. 5). 


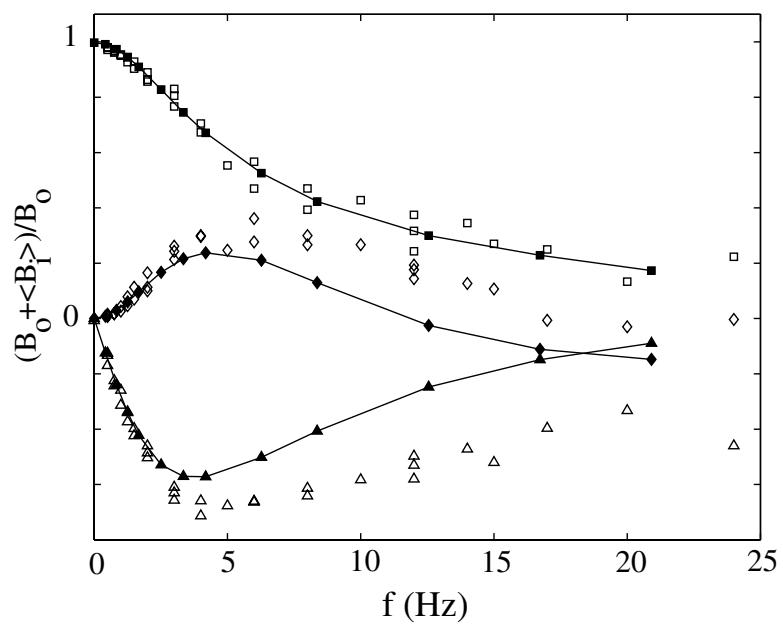

FIG. 5. Components of the total mean magnetic field as a function of the rotation frequency of disk (2). The disk radius is $R=190 \mathrm{~mm}$ with curved blades. There are no baffles on the inner wall of the cylindrical vessel. The magnetic field is measured or computed at $z=100 \mathrm{~mm}$. The open symbols refer to the measurements and the dark ones to numerical results obtained from the computation of the induced magnetic field by the mean flow alone $\left[(\bullet) \frac{\left\langle B_{x}\right\rangle}{B_{o}} ;(\boldsymbol{\square}) \frac{B_{o}+\left\langle B_{y}\right\rangle}{B_{o}} ;(\boldsymbol{\Delta}) \frac{\left\langle B_{z}\right\rangle}{B_{o}}\right]$.

Using dimensional analysis, we can write $\left\langle B_{x}(\vec{r})\right\rangle=$ $B_{0} \mathcal{F}\left(\vec{r}, R_{m}, P_{m}, N\right)$. As said above, for $B_{0}$ small enough, $\mathcal{F}$ does not depend on the interaction parameter $N$. We have provided a detailed investigation on the nonlinear behavior of $\mathcal{F}$ on $R_{m}$ even at small $R_{m}$, in a nongeometrically constrained flow. Its dependence on $P_{m}$, or equivalently on the Reynolds number of the flow, is governed by the relative contribution of the two nonlinear source terms $\vec{\nabla} \times(\langle\vec{V}\rangle \times\langle\vec{B}\rangle)$ and $\vec{\nabla} \times(\langle\vec{v} \times \vec{b}\rangle)$ in Eq. (3). Both the velocity field, measured in water [5], and the magnetic field display large fluctuations (roughly $20 \%)$. An estimate of the effect of $\langle\vec{v} \times \vec{b}\rangle$ can be obtained from the difference between the measured magnetic field (open symbols in Fig. 5) and a numerical computation of the magnetic field induced by the mean flow alone (solid symbols) measured in a $1 / 2$-scale water experiment as explained in [18]. We observe a perfect agreement at low $R_{m}$ (up to nearly $f=5 \mathrm{~Hz}$ ). At larger $R_{m}$, Fig. 5 displays clear differences between the computed and measured fields for $\left\langle B_{x}\right\rangle$ and $\left\langle B_{z}\right\rangle$. Although we cannot rule out possible inaccuracies of the numerical modelization (the cylindrical boundary made of copper is modeled by sodium at rest, the boundary conditions are periodic along the $x$ axis, slight nonaxisymmetry of the mean flow is not taken into account), we observe a clear difference between the magnetic induction by the turbulent flow at large $R_{m}$ and the computed one resulting from the mean flow alone.

We acknowledge the assistance of J.-B. Luciani and M. Moulin. We thank J. Léorat for letting us use his numerical code to get the curves displayed in Fig. 5.
This work is supported by the French institutions Direction des Sciences de la Matière and Direction de l'Energie Nucléaire of CEA, Ministère de la Recherche, and Centre National de Recherche Scientifique. J. Burguete was supported by a grant from ministerio de Educación y Ciencia (Spain) while at CEA-Saclay.

*Present address: Departamento de Física y Matemática Aplicada, Universidad de Navarra, E-31080 Pamplona, Spain.

[1] Ya. B. Zeldovich, A. A. Ruzmaikin, and D. D. Sokoloff, Magnetic Fields in Astrophysics (Gordon and Breach, New York, 1983).

[2] See, for instance, L. Garzotti et al., Phys. Rev. Lett. 84, 5532 (2000); P.W. Fontana et al., Phys. Rev. Lett. 85, 566 (2000), and references therein.

[3] B. Lehnert, Ark. Fys. 13, 109 (1957).

[4] P. Odier, J.-F. Pinton, and S. Fauve, Phys. Rev. E 58, 7397 (1998).

[5] M. Bourgoin et al., Phys. Fluids 14, 3046 (2002).

[6] M. Steenbeck et al., Sov. Phys. Dokl. 13, 443 (1968).

[7] E. N. Parker, Astrophys. J. 122, 293 (1955).

[8] F. Krause and K.-H. Rädler, Mean Field Magnetohydrodynamics and Dynamo Theory (Pergamon Press, New York, 1980).

[9] H. K. Moffatt, Magnetic Field Generation in Electrically Conducting Fluids (Cambridge University Press, Cambridge, 1978).

[10] P. H. Roberts, in Lectures on Solar and Planetary Dynamos, edited by M. R. E. Proctor and A. D. Gilbert (Cambridge University Press, Cambridge, 1994), Chap. 1.

[11] R. Stieglitz and U. Müller, Phys. Fluids 13, 561 (2001); A. Gailitis et al., Phys. Rev. Lett. 86, 3024 (2001).

[12] See, for instance, L. Marié et al., Dynamo and Dynamics, a Mathematical Challenge, edited by $\mathrm{P}$. Chossat et al. (Kluwer Academic Publishers, Dordrecht, 2001), pp. 35-50.R. O'Connell et al., pp. 5966, W. L. Shew et al., pp. 83-92.

[13] A. B. Reighard and M. R. Brown, Phys. Rev. Lett. 86, 2794 (2001).

[14] A. Lanotte, A. Noullez, M. Vergassola, and A. Wirth, Geophys. Astrophys. Fluid Dyn. 91, 131 (1999); V. A. Zheligovsky, O. M. Podvigina, and U. Frisch, Geophys. Astrophys. Fluid Dyn. 95, 227 (2001).

[15] R. L. Parker, Proc. R. Soc. London, Ser. A 291, 60 (1966); N. O. Weiss, Proc. R. Soc. London, Ser. A 293, 310 (1966).

[16] P. Odier, J.-F. Pinton, and S. Fauve, Eur. Phys. J. B 16, 373 (2000).

[17] K.-H. Rädler, E. Apstein, and M. Schüler, in Transfer Phenomena in Magnetohydrodynamic and Electroconducting Flows (Kluwer Academic Publishers, Dordrecht, 1999), Vol. 1, pp. 9-14.

[18] L. Marié, J. Burguete, F. Daviaud, and J. Léorat, "Numerical Study of Homogeneous Dynamo Based on Experimental von Kármán Type Flows" (to be published). 